\title{
La conformación de redes en los procesos de Inclusión educativa de niños con Trastornos del Desarrollo.
}

\author{
The conformation of networks in the processes \\ of Educational Inclusion of children \\ with Developmental Disorders.
}

Ana C. Isaias.

Facultad de Ciencias Médicas. Universidad Nacional de Rosario. Rosario; Argentina.

Autor por correspondencia: Ana C. Isaías - anaclara.isaias@unr.edu.ar

Conflicto de intereses: no presenta.

\section{Resumen}

Las redes son formas de interacción social, definidas por intercambios dinámicos entre los sujetos que las forman; en este caso, abordaremos aquellas que intentan dar respuesta a las necesidades de inclusión educativa de niños con Trastornos del Desarrollo (TD), asumiéndolas como estrategias que permiten potenciar recursos y contribuir a la resolución de problemas. Se indaga qué actores, instituciones, obstáculos y ayudas surgen en los procesos inclusivos. Para ello, se diseñó un estudio exploratorio y transversal, apelando a la estrategia de triangulación; por medio de una entrevista semiestructurada. Formaron parte de la población 14 profesionales de la salud que atienden niños con TD, en el Hospital de Niños Zona Norte. Los actores mencionados con mayor frecuencia son: el psicólogo (12), el fonoaudiólogo (8) y el neuropediatra (6) y el terapista ocupacional (6). Las instituciones más nombradas como formantes de la red son la escuela común (11) y la especial (11). En relación a los obstáculos, 2 entrevistados no mencionan ninguno, 4 detectan obstáculos actitudinales; 3 comunicativos; 2 administrativos, 2 epistemológicos y 1 comunicativos y administrativos. Por ejemplo: "Falta de comunicación, poca disponibilidad de horarios para reuniones". Por otra parte; 9 destacan beneficios para los usuarios, 1 de ellos los ubica en relación a los profesionales y 4 mencionan a ambos. Por ejemplo, "Procesos de integración con mejores resultados en cuanto a permanencia, experiencia y aprendizaje de todos los actores". Si bien la muestra seleccionada es pequeña, podemos reflexionar acerca de las estrategias de los equipos, dirigidas a ofrecer una mejor atención y aportar al proceso de inclusión escolar, desbordando los límites de los consultorios, entramándose en diversos sectores que redundan en experiencias enriquecedoras.

Palabras claves: Trastornos del Desarrollo. Inclusión educativa. Equipos de salud. Redes. Interdisciplina.

\begin{abstract}
Networks are forms of social interaction, defined by dynamic exchanges among the subjects that form them. In this case, we will study those that try to respond to the educational inclusion needs of children with Developmental Disorders (DD), assuming them as strategies that allow to enhance resources and contribute to problem solving. It analyses the actors, institutions, obstacles and
\end{abstract}


aid that arise in inclusive processes. To achieve this, it was selected an exploratory and crosssectional study, appealing to the triangulation strategy; through a semi-structured interview. The population comprised 14 health professionals who care for children with DD, at the "Zona Norte" Children Hospital. The most frequently mentioned actors are: psychologist (12), speech therapist (8) and neuropediatrician (6) and occupational therapist (6). The institutions most named as forming the network are the common school (11) and special school (11). Results shows that 2 of the interviewed did not mention any obstacles, 4 of them detected attitudinal obstacles; 3 interviewed mentioned communicative obstacles; 2 of them mentioned administrative or epistemological obstacles, and 1 interviewed detected communicative and administrative obstacles. For example: "Lack of communication, little availability of meeting times." On the other hand; 9 highlight benefits for users, 1 of them places them in relation to professionals and 4 mention both. For example, "Integration processes with better results in terms of permanence, experience and learning of all the actors". Although the selected sample is small, we can reflect on the strategies of the teams, aimed at offering better care and contributing to the process of school inclusion, exceeding the limits of the clinics, entering various sectors that result in enriching experiences.

\section{Keywords:DevelopmentalDisorders—educationalinclusion—healthteams—networks- interdisciplinary}

\section{Introducción}

Este trabajo tiene como antecedente el estudio "Interdisciplina y articulación de los equipos de educación y salud en el Proceso de Inclusión de niños con Trastornos del Desarrollo en la Escuela Común”, en el cual se indagó acerca de las experiencias vinculadas al proceso de inclusión educativa desde la mirada docente. En las apreciaciones de los docentes acerca de su participación en procesos inclusivos de niños y niñas con Trastornos del Desarrollo (TD), parecen entreverse demandas al Sistema Educativo. Mientras que los docentes de enseñanza común demandan apoyo de la institución y la participación de docentes integradores, los docentes especiales sugieren que falta ayuda institucional. Entre sus decires se destacan: "se hicieron adaptaciones espontáneas" "estuve muy aislada en la toma de decisiones". 5 de los 11 docentes encuestados no percibieron obstáculos al momento de incluir al niño, sin embargo, manifiestan haberse sentido solos o haber "cargado" con la integración. En este punto, 6/11 docentes mencionan obstáculos administrativos, edilicios, curriculares y de formación. Entre las menciones de los entrevistados citamos las siguientes: "tuve que cargar con la integración" "la maestra integradora iba solo algunos días". La necesidad de formación específica en la problemática de los TD, aparece tanto en el discurso de docentes de grado como en el de maestras integradoras (5/11). Entre las ayudas que percibieron los docentes entrevistados, mencionan la participación familiar y la presencia de equipos de profesionales. Finalmente están quienes plantean que existen instituciones más "abiertas" o "cerradas". La sistematización de experiencias de inclusión de niños con TD es escasa, aunque sabemos que existen muchas que han sido exitosas (1).

Las conclusiones del mismo invitaron a profundizar algunos aspectos del proceso de Inclusión en niños que presentan condiciones de TD. En ese sentido se avanza en la perspectiva de los profesionales de la salud, en el marco del trabajo "Inclusión de niños con Trastornos del Desarrollo en la Escuela Común, aproximaciones desde la mirada de los profesionales de la Salud". En una primera etapa se indagó acerca de los actores del sistema educativo con los que mantuvieron relación los profesionales, las características de la comunicación mantenida por el equipo y su frecuencia (2). En este caso, interesa indagar qué obstáculos y/o beneficios surgen en la conformación de redes, donde se articulan miradas provenientes de las esferas educativa y sanitaria, desde la perspectiva de los profesionales de la salud.

En relación a los procesos de Inclusión en la Escuela Común, éstos constituyen fenómenos muy complejos, que implican una política educativa que atienda a la diversidad. En esta lógica, participan multiplicidad de actores que provienen del campo educativo y también del campo de la salud, desde diferentes áreas del conocimiento y especialidades. Las trayectorias educativas de niños y niñas con TD en la escuela común, constituyen procesos de búsqueda constantes de mejores maneras de responder a la diversidad del alumnado. Se trata de aprender a vivir con la diferencia y a la vez de valorarla. (3)

Cuando pensamos la complejidad lo hacemos desde la perspectiva de Morín (1990); quien define la complejidad como “...un tejido (complexus: lo que está tejido en conjunto) de constituyentes heterogéneos inseparablemente asociados: presenta la paradoja de lo uno y lo múltiple..." (4) 
Tomamos aquí la definición de TD de Baro Tizón (5), como alteraciones, disfunciones o dificultades que afectan la capacidad adaptativa de una persona que conlleva a necesidades de apoyo y recursos en el contexto en que se desenvuelve. Esto no significa tomarlas como marcas que lo excluyen, sino como un rasgo distintivo más de su subjetividad que puede aportar al aprendizaje (6).

Resulta desafiante interpelar tanto al sistema educativo como al de salud en vistas de identificar prácticas cotidianas que den cuenta del accionar de los mismos al momento de articular acciones conjuntas que permitan construir estrategias habilitantes, que potencien la constitución subjetiva y el aprendizaje de los niños que transitan los espacios escolares. Por ello, nos interesa indagar acerca de la conformación de redes que se construyen para sostener la asistencia de los niños con TD ya que constituye un abordaje indispensable en las problemáticas infantiles.

“... las redes son, antes que nada, formas de interacción social, espacios sociales de convivencia y conectividad. Se definen fundamentalmente por los intercambios dinámicos entre los sujetos que las forman. Las redes son sistemas abiertos y horizontales, y aglutinan a conjuntos de personas que se identifican con las mismas necesidades y problemáticas" (7).

En este caso, abordaremos las redes que se constituyen para dar respuesta a las necesidades de inclusión educativa de niños con problemáticas en su desarrollo, asumiendo a las redes como estrategias que permiten a un grupo de personas potenciar sus recursos y contribuir a la resolución de problemas. Estas tienen la lógica de organizar a la sociedad en su diversidad, mediante la estructuración de vínculos entre grupos con intereses y preocupaciones comunes, con un desafío a la estructura piramidal, vertical, de la organización social y proponen una alternativa a esta forma de organización que pueda hacer frente a las situaciones de fragmentación y desarticulación que se vive en la actualidad.

La articulación al interior del sistema de Salud, así como las interacciones interinstitucionales, parecen constituir una buena práctica en el abordaje terapéutico y educativo de los niños con desafíos en su desarrollo. Estas conexiones exceden el sistema de salud, y se intrincan con otros sectores, para articular información, cooperar, coordinar e integrar acciones destinadas a mejorar las condiciones de salud de la población. Sin embargo, la fragmentación inherente al Sistema Sanitario y la dificultad en articular acciones intersectoriales se manifiestan como obstáculos en las prácticas de los profesionales que asisten a estos usuarios.

Entendemos intersectorialidad como la intervención coordinada de instituciones representativas de más de un sector social, en acciones destinadas, total o parcialmente, a tratar los problemas vinculados con la salud, el bienestar y la calidad de vida (8).

Estas articulaciones avanzan mediante la superación de obstáculos; es decir, entorpecimientos, confusiones, causas de estancamiento y hasta de retroceso e inercia que impiden al sujeto avanzar en diversas tareas. (Bachelard, 1974) Si bien este autor se refiere a los obstáculos epistemológicos inherentes al proceso del conocimiento científico, consideramos que aplica adecuadamente al caso del trabajo en equipo, ya que en nuestras prácticas profesionales cargamos con las ideas, concepciones, conocimientos cotidianos y prácticos de nuestra época, de nuestra cultura, de nuestra clase social que actúan como prejuicio. (9)

No podemos pensar semejantes articulaciones sin mencionar un concepto clave, el de interdisciplina.

"La interdisciplinariedad es un posicionamiento, no una teoría unívoca. Ese posicionamiento obliga básicamente a reconocer la incompletud de las herramientas de cada disciplina. Legitima algo que existía previamente: las importaciones de un campo a otro, la multireferencialidad teórica en el abordaje de los problemas y la existencia de corrientes de pensamiento subterráneas ——de épocaatravesando distintos saberes disciplinarios". (10)

La misma autora plantea que la actividad interdisciplinaria se inscribe en la acción cooperativa de los sujetos, requiere por tanto de espacios y tiempos compartidos para poder pensar en conjunto intervenciones posibles. Por ello interesa conocer cómo se configuran estas redes desde la perspectiva de los profesionales de salud de un efector público con 2do y $3 e r$ nivel de complejidad. 


\section{Materiales y Métodos}

Esta investigación es aplicada y descriptivo- exploratoria. Para llevarla a cabo se recurrió al diseño transversal, de corte mixto (cuali- cuantitativo). En primera instancia se solicitó autorización al Director del hospital y por su intermedio al comité de Docencia e Investigación del Efector, que autorizó el acceso a los profesionales para ser entrevistados. Los datos de la investigación se recogieron por medio de entrevistas semiestructuradas, a los fines de recoger la complejidad de las situaciones estudiadas. El instrumento se elaboró contemplando preguntas cerradas y abiertas. Las respuestas a las preguntas vinculadas a qué profesionales e instituciones participaron del trabajo en red, fueron trabajadas de manera cuantitativa. Mientras que las respuestas vinculadas a obstáculos y beneficios, fueron codificadas en categorías o grupos de respuestas con convergencias semánticas; y luego de volcarlas en los gráficos, algunas de ellas han sido recuperadas como ejemplos para conservar la riqueza cualitativa de los dichos de los entrevistados.

La población de estudio estuvo conformada por 14 profesionales de la salud del Hospital de Niños Zona Norte (efector de 2do y 3er nivel de complejidad) al que asisten usuarios con diagnóstico de TD. Accedieron a ser entrevistadas: 2 neuropediatras, 1 médica pediatra, 2 licenciadas en fonoaudiología, 1 músicoterapeuta, 1 terapeuta ocupacional, 2 kinesiólogas, 1 docente especial y 4 trabajadoras sociales.

\section{Resultados y discusión}

Tal como se observa en la tabla $n^{\circ} 1$, existe gran variedad en la constitución de los equipos en la intervención de niños con TD. El funcionamiento de un equipo de salud requiere de la planificación de actividades dentro de un dispositivo de trabajo que incluya los tiempos destinados a discusión, ateneos y reuniones a los fines de sostener un posicionamiento interdisciplinario. (11)

Tabla 1. Actores que participaron de las redes de trabajo en la inclusión de niños con TD en la escuela común, según la perspectiva de profesionales de la salud del Hospital de Niños Zonal Norte de Rosario, 2019.

\begin{tabular}{|l|c|}
\hline Actores que participaron del equipo & \multicolumn{1}{|c|}{ Frecuencia } \\
\hline Psicólogo & 12 \\
\hline Fonoaudiólogo & 8 \\
\hline Neuropediatra & 7 \\
\hline Terapista Ocupacional & 7 \\
\hline Médico pediatra & 6 \\
\hline Psicopedagogo & 6 \\
\hline Psiquiatra infantil & 5 \\
\hline Otros & 5 \\
\hline Musicoterapeuta & 5 \\
\hline Kinesiólogo & 4 \\
\hline Estimulador Temprano & 4 \\
\hline Médico Generalista & 2 \\
\hline Total & 1 \\
\hline
\end{tabular}


Estas acciones conjuntas se imbrican en un entramado de constituyentes heterogéneos indisolublemente asociados, que nos permiten construir diversos modos de pensar interprácticas profesionales. Es decir que, en la composición de las redes observamos no solo la interdisciplina, sino también la interinstitucionalidad, ya que se establecen conexiones entre diferentes niveles y sectores del Sistema de Salud; pero además se establecen nexos intersectoriales; implicando acciones coordinadas de instituciones representativas de más de un sector social, en actuaciones destinadas a sostener el proceso de inclusión de los niños con TD en su trayecto por la escuela común.

La Figura 1 muestra que 11 de los 14 profesionales manifiestan que las instituciones implicadas en el abordaje de niños con TD son la escuela común y la especial. 5 mencionan el Centro de Salud de ler nivel de atención, 5 de ellos mencionan el Centro Educativo Terapéutico, 4 de los entrevistados nombran los Centros de Acción Familiar, y 5 destacan la participación de Centros de Estimulación Temprana, 2 otros Hospitales y 1 menciona vecinales.

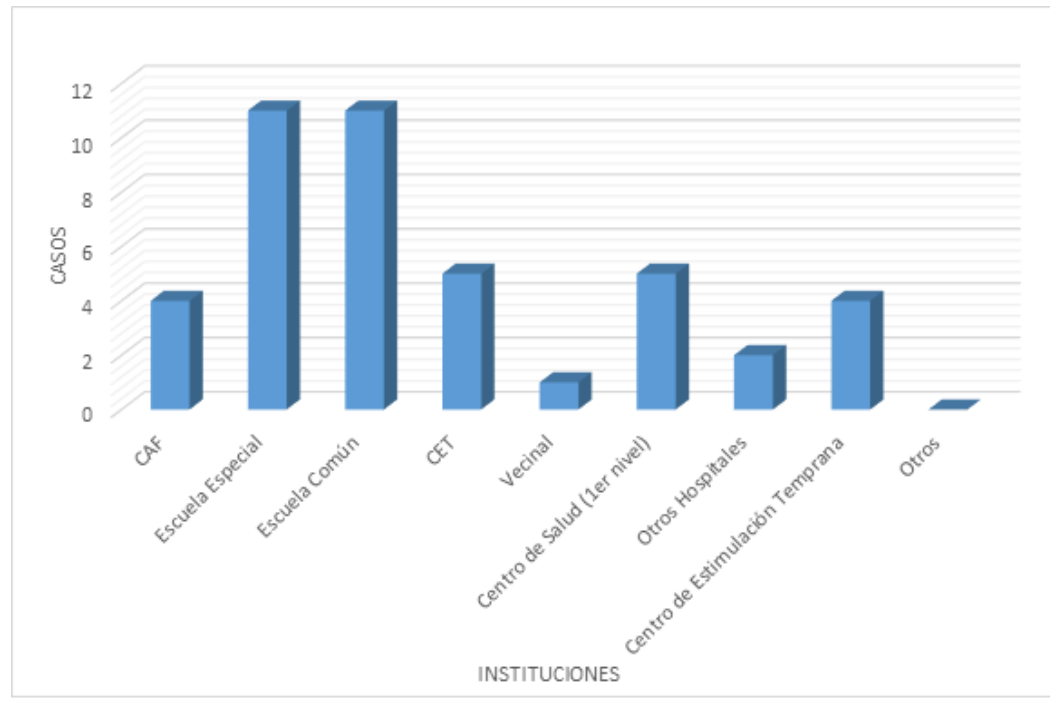

Figura 1: Instituciones que participaron de las redes de abordaje de niños con TD, desde la perspectiva de profesionales de la salud del Hospital de Niños Zona Norte de Rosario, 2019. CAF: Centro de acción familiar; Escuela Esp: Escuela Especial; Escuela Com: Escuela Común; CET: Centro educativo terapéutico; CS: Centro de salud; Otros Hosp: Otros Hospitales; Centro Est Temp: Centro de estimulación temprana.

Cuando configuramos redes, intentamos proveer servicios de salud integrales a la población, de forma equitativa, con el objetivo de promover, preservar y/o recuperar la salud de las personas (12) por lo que estos intercambios pueden pensarse como estrategias intersectoriales (13).

En el trabajo en red, podemos identificar una serie de obstáculos en las prácticas profesionales, que devienen de nuestras propias concepciones; o bien de la complejidad institucional y social en que se enmarcan dichas acciones. (14)

Como puede observarse en la Figura $n^{\circ}$ 2, 2 de los profesionales no mencionan obstáculos en el trabajo en red; 4 de ellos perciben dificultades actitudinales; 3 de ellos mencionan obstáculos comunicativos; 2, administrativos; 2 mencionan obstáculos epistemológicos; 1 comunicativos y administrativos. 


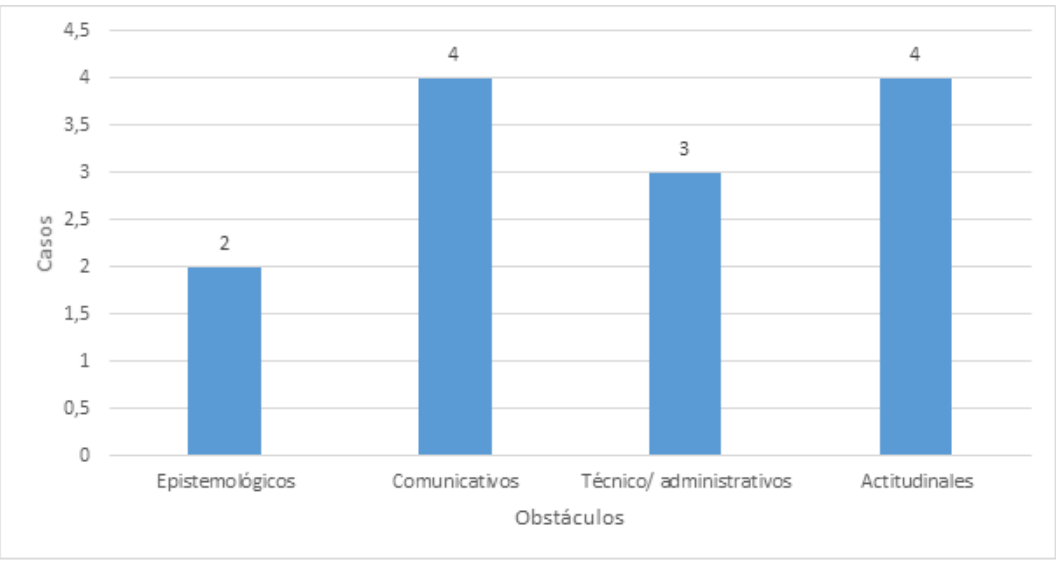

Figura 2: Obstáculos percibidos durante el trabajo en red por parte de los profesionales del Hospital de Niños Zona Norte de Rosario, 2019.

Para ejemplificar la categoría de obstáculos epistemológicos se cita la respuesta (entrevista $n^{\circ}$ 5) que reza "Los obstáculos que se perciben son distintos posicionamientos que hay entre los profesionales respecto del tema TD y dificultad de poder aunar criterios".

Respecto a la categoría comunicativos se cita la respuesta $n^{\circ} 9$ que menciona "La falta de comunicación entre todos los que forman parte de la red".

En relación a la categoría técnico/ administrativa, un ejemplo paradigmático lo constituye la respuesta $n^{\circ} 1$ que responde como obstáculo "Tiempo disponible".

Como obstáculos actitudinales, mencionan por ejemplo, "La disponibilidad de los diferentes actores frente al compromiso con el niño, depositando la dedicación en unos pocos. La continuidad del abordaje (principalmente el no involucramiento de los médicos y especialistas)." (Entrevista 13).

Sabemos que más allá de las limitaciones del trabajo en red, podemos percibir una serie de beneficios, en tanto actividad horizontal y participativa orientada a la solución de diferentes problemáticas. Todos los entrevistados reconocen que el trabajo en red presenta ventajas. En este sentido el aporte del funcionamiento en redes, personales, grupales, institucionales, se constituye en un soporte para dar respuestas. Por ello, es tan importante la construcción de lazos con todas las instituciones y organizaciones de la comunidad. (15)

En relación a los beneficios del trabajo en red, tal como vemos en la Figura ${ }^{\circ} 3,9$ de los profesionales mencionan las ventajas que tiene el trabajo en red para el usuario del servicio de salud, por ejemplo "Mayor adherencia al tratamiento de padres y niños". (Entrevista $\left.n^{\circ} 10\right) .1$ rescata los beneficios que tiene para los trabajadores de la salud, como lo expresa la Entrevista $n^{\circ} 9$ "Aportar distintas miradas del paciente en distintos contextos, unificar criterios de abordaje". Finalmente, 4 de los profesionales, mencionan en sus respuestas beneficios tanto para los usuarios como para los profesionales implicados en el trabajo en red. La entrevistada $\mathrm{n}^{\circ} 3$ expresa "Procesos de integración con mejores resultados en cuanto a permanencia, experiencia y aprendizaje de todos los actores".

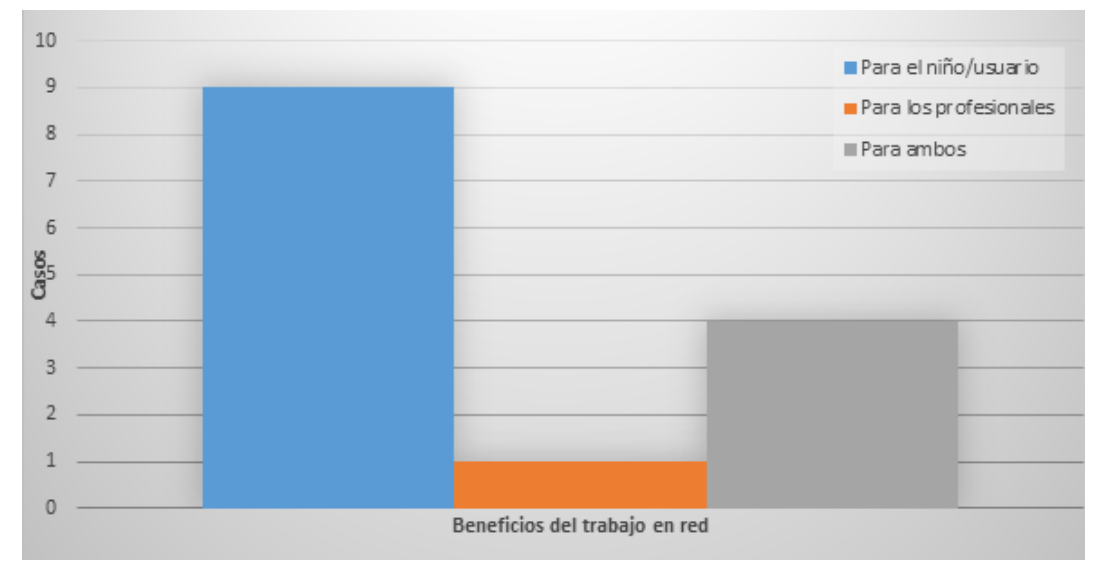

Figura 3: Beneficios del trabajo en red según los profesionales de la salud que atienden a niños con TD del Hospital de Niños Zona Norte de Rosario, 2019. 


\section{Conclusiones}

Aunque la muestra seleccionada para este trabajo no permite establecer generalizaciones, sí nos habilita a reflexionar acerca de las prácticas construidas por los equipos de salud en beneficio de la población atendida con TD, a los fines de aportar al proceso de inclusión escolar. Estas praxis desbordan los límites de los consultorios, se entraman en la institución hospitalaria; pero también se vinculan a diversos sectores y actores educativos, sociales, de la sociedad civil, etc. generando experiencias enriquecedoras para los usuarios del sistema de salud y para todos los actores implicados en este proceso.

Fuente de financiamiento: no presenta.

\section{Referencias Bibliográficas}

1. Trabajo presentado en las XIII Jornadas de Comunicación científica de la Escuela de Fonoaudiología, 28 de mayo de 2015.

2. Isaias, A. Inclusión de niños con TD en la Escuela Común, aproximaciones desde la mirada de los profesionales de la salud. Libro de resúmenes. Área de Ciencia Tecnología e Innovación para el desarrollo CyT XIII, Rosario: UNR; 2019.

Aiscow, M. y Echeita, G. La Educación inclusiva como derecho. Marco de referencia y pautas de acción para el desarrollo de una revolución pendiente. file://C:/Users/ASYT/Downloads/Dialnet_LaEducacionInclusivaComoDerecho-3736956.pdf (2/10/2020)

Morín, E. Introducción al pensamiento complejo, Buenos Aires: Gedisa; 1990.

9. FLACSO. Informe Técnico para el Diseño de Plan de Trabajo para reorientación de Programas de Salud Pública, Chile: Facultad Latinoamericana de Ciencias Sociales; 2015.

10. Bachelard, G. La formación del espíritu científico, Buenos Aires: Siglo XXI; 1974.

11. Stolkiner, A. IX Jornadas Nacionales de Salud Mental. I Jornadas Provinciales de Psicología, Salud Mental y Mundialización: Estrategias Posibles en la Argentina de hoy. 7 y 8 de octubre, Posadas; 2005 Solitario, R.; Garbus, P. y Stolkiner, A. Atención primaria de la salud e interdisciplina. Revista de la Asociación Médica de Bahía Blanca. 2007; $\operatorname{Vol} 17, n^{\circ} 3: 63-68$.

13. OPS. La renovación de la Atención Primaria de la salud en las Américas. Redes integrales de servicios de salud, Washington DC: OPS; 2010.

14. Serrano Gallardo, M. Intersectorialidad, la clave para enfrentar las Desigualdades Sociales en Salud. Revista Latino—Am. Enfermería, 2019; 27(e3124.): 1-2.

15. Días, P,; Herniques, P.; et al. Desafios da intersetorialidade nas políticas públicas: o dilema entre a suplementação nutricional e a promoção da alimentação saudável em escolas. Cadernos de Saúde Pública, 2019; 34 (12), e00035218: 1-13.

16. Presidencia de la Nación. Curso en Salud Social y Comunitaria, Buenos Aires; s/d. http://iah.salud.gob.ar/doc/Documentol82.pdf $(2 / 10 / 2020)$ 